J3eA, Journal sur l'enseignement des sciences et technologies de l'information et des systèmes, Volume 4, Hors-Série 2, 32 (2005)

DOI : http://dx.doi.org/10.1051/bib-j3ea:2005732

(C) EDP Sciences, 2005

\title{
Réalisation d'un RADAR de poursuite
}

B. Mouton, F. Vincent et C. Nouals

Département Avionique et Systèmes

ENSICA

1, place Emile BLOUIN

F-31056 Toulouse, France 


\title{
Réalisation d'un RADAR de poursuite
}

\author{
B. MOUTON, Département Avionique et Systèmes, ENSICA, 1, Place Emile BLOUIN, \\ 31056 Toulouse.mouton@ensica.fr \\ F. VINCENT, Département Avionique et Systèmes, ENSICA, 1, Place Emile BLOUIN, \\ 31056 Toulouse. vincent@ensica.fr \\ C. NOUALS, Département Avionique et Systèmes, ENSICA, 1, Place Emile BLOUIN, \\ 31056 Toulouse. nouals@ensica.fr
}

\section{Résumé :}

Le Département Avionique et Systèmes de l'ENSICA (Ecole Nationale Supérieure d'Ingénieurs de Constructions Aéronautiques) propose à ses élèves de $1^{\text {ère }}$ Année des projets d'une durée de 20 heures au cours desquels les élèves doivent faire preuve d'autonomie et d'initiative.

De manière à faire apparaître les liens entre les différentes disciplines de base de l'enseignement EEA, nous proposons aux étudiants de réaliser un système ultrasonore permettant de suivre un émetteur actif et de calculer sa distance (Radar de poursuite).

Au cours de ce projet les élèves doivent acquérir des connaissances sur le principe de fonctionnement des RADARS ainsi que sur les capteurs ultrasonores, sur les antennes et sur les moteurs à courant continu. Ils doivent aussi faire des mesures sur les capteurs et l'antenne afin de réaliser des amplificateurs, le circuit pour l'estimation de l'angle de dépointage, l'amplificateur correcteur pour commander le moteur et enfin un circuit pour calculer la distance cible - radar.

Mots clés : Projet pédagogique, électronique, ultrason, antenne, radar, mesure de distance.

\section{Introduction}

L'ENSICA (Ecole Nationale Supérieure d'Ingénieurs de Constructions Aéronautiques) forme des ingénieurs pluridisciplinaires aptes à conduire, à terme, des projets de systèmes complexes essentiellement dans le secteur aéronautique et spatial. Les élèves ingénieurs reçoivent, entre autre, une formation de base couvrant le spectre de l'enseignement EEA (électronique, systèmes temps réels, automatique, traitement du signal, électromagnétisme). De manière à faire apparaître les liens entre ces différentes disciplines de base, nous proposons aux étudiants de réaliser un système ultrasonore permettant de suivre un émetteur actif et de calculer sa distance (RADAR de poursuite).

\section{Description du projet 2.1 Généralités}

Le but donné aux étudiants est de réaliser l'électronique (analogique) d'un système capable de rester pointé vers un émetteur ultrasonore et de calculer la distance de cet émetteur. Pour cela les étudiants disposent d'un moteur à courant continu, de 2 récepteurs ultrasonores (large bande autour de $40 \mathrm{KHz}$ ), d'un réflecteur parabolique, de plaques de test électronique. Pour des raisons de simplicité, le suivi de l'émetteur ne se fait que suivant la direction horizontale (un seul axe de rotation).

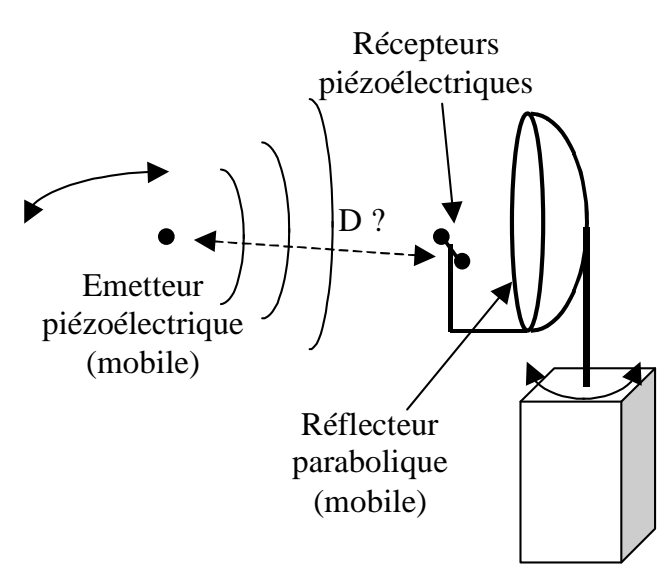

De nombreux systèmes RADAR utilisants le même principe de suivi sont en fonctionnement dans le monde («RADAR de tracking »). Nous proposons donc aux étudiants d'utiliser un système de traitement analogue à ces systèmes réels.

\subsection{Mesure de l'angle de dépointage et correction}

Le calcul de l'angle de dépointage (angle entre la source ultrasonore et l'axe de symétrie de l'antenne) est basé sur une comparaison des amplitudes des signaux reçus sur chacun des récepteurs. En effet, si l'émetteur est dans l'axe de l'antenne les 2 récepteurs reçoivent le même signal en amplitude. Par contre, du fait du diagramme d'antenne lié au réflecteur parabolique, si l'antenne est dépointée, on observera une dissymétrie sur les amplitudes des signaux reçus. 


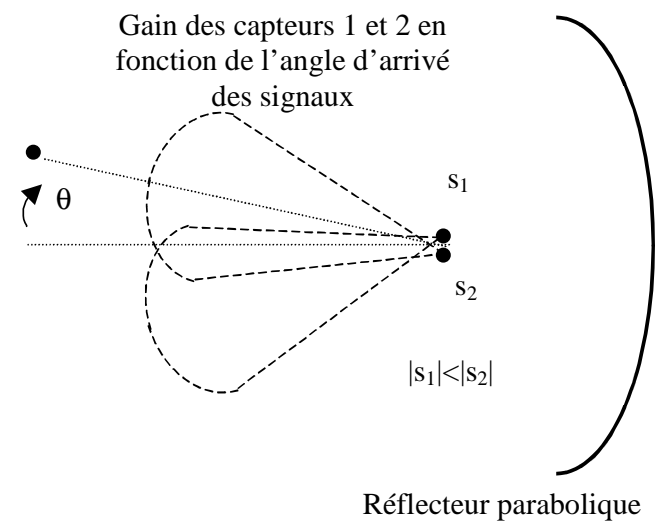

L'information qui va nous permettre de commander l'antenne est donc $\left|s_{1}(t)\right|-\left|s_{2}(t)\right|$. Cependant, pour nous affranchir des problèmes d'amplitude liés à la distance et à la puissance de l'émetteur, nous préférons utiliser le rapport $\frac{\left|s_{1}(t)\right|-\left|s_{2}(t)\right|}{\left|s_{1}(t)\right|+\left|s_{2}(t)\right|}$ pour commander l'antenne. Il est possible de montrer que ce rapport est un estimateur de l'angle de dépointage $\theta$ quand celui-ci est faible.

\subsection{Mesure de la distance cible-RADAR}

La distance entre l'émetteur et le Radar étant faible (quelques mètres), il est très difficile d'utiliser le principe classique des radars impulsionnels qui consiste à mesurer le temps mis par une impulsion sonore pour atteindre le Radar. Nous préférons utiliser ici une méthode plus précise pour la mesure de courte distance. Cette méthode est en particulier utilisée pour les Radars anti-collision dans l'automobile. Contrairement aux Radars impulsionnels, l'onde ultrasonore est émise de façon continue. Celle-ci est modulée linéairement en fréquence. Le retard de propagation entre l'onde émise et l'onde reçue peut donc être mesuré par la différence de fréquence entre ces deux ondes. Cette différence est constante (pour une distance donnée) comme le montre la figure ci-dessous qui représente l'évolution de la fréquence instantanée en fonction du temps.

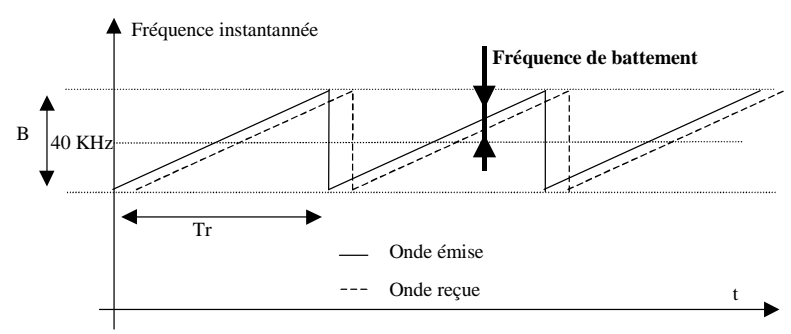

D'après ce schéma, on peut déduire que la fréquence de battement $F_{b}$ est proportionnelle au retard de propagation $\tau$. Ce dernier étant égal au rapport de la distance parcourue sur la célérité de l'onde sonore, on obtient la relation suivante entre la fréquence de battement et la distance recherchée $D$ :

$$
F_{b}=\frac{B}{T_{r}} \frac{D}{c}
$$

Le choix de la bande $B$ et de la période de répétition de l'onde $T_{r}$ est laissé aux élèves. Celui-ci influe sur l'ambiguité et la résolution du système.

\subsection{Schéma fonctionnel}

En fonction des informations précédentes, nous proposons aux élèves de réaliser un système RADAR d'après le schéma fonctionnel suivant :

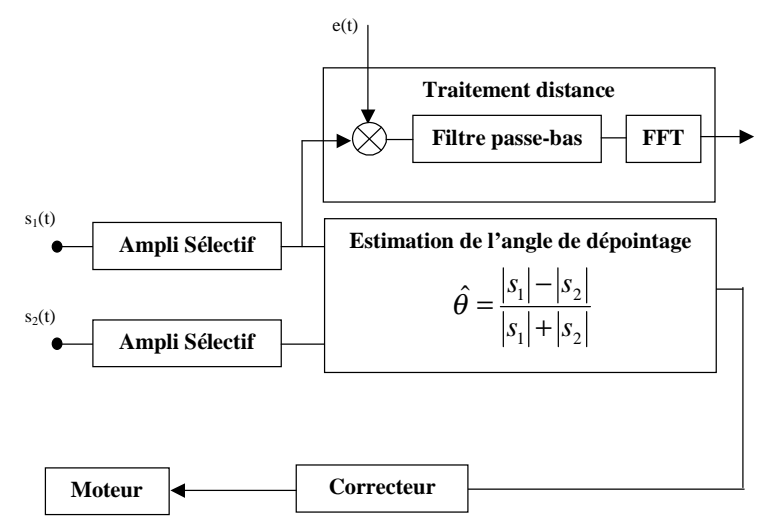

\section{Démarche pédagogique \\ 3.1 déroulement des séances}

Pour ce projet de 20 heures les élèves travaillent en groupe de 6. En général, ils se répartissent les taches comme suit :

- 2 élèves développent et testent les amplificateurs de réception accordés sur la fréquence porteuse.

- $\quad 2$ élèves développent et testent l'estimation angulaire et la correction associée

- 2 élèves développent et testent la mesure de distance.

Tous doivent cependant travailler ensemble pour définir les paramètres caractéristiques de la forme d'onde (bande, période de répétition, ....).

D'un point de vue pratique, les 20 heures sont découpées en 8 séances de 2 heures 30 . La moitié de celle-ci sont encadrées par un enseignant, l'autre moitié correspond à des créneaux horaires libres. Un support technique est toutefois présent durant toutes les séances.

A l'issu des 20 heures, les élèves doivent rédiger un rapport technique d'une dizaine de pages. Celui-ci doit résumer la démarche employée et justifier par 
des mesures les performances de leur système (précision en distance, ..).

Ils doivent, en outre présenter leur travail devant un jury d'enseignants de l'école au cours d'un oral de 30 minutes.

La note finale tient compte des résultats atteints ainsi que du rapport technique et de la soutenance.

\subsection{Matériel utilisé}

L'équipe d'enseignants donne à chaque groupe d'élèves un réflecteur parabolique d'un diamètre de 12 centimètres, un émetteur et 2 récepteurs ultrasonores à large bande $(10 \mathrm{KHz}) \mathrm{de}$ fréquence centrale $40 \mathrm{KHz}$, d'un moteur à courant continu et de plaquettes d'essai (labdec).

Les élèves sont totalement libres dans le choix des circuits à mettre en œuvre. C'est pourquoi ils ont accès à tous les composants électroniques « classiques» (résistances, capacités, diodes, transistor, ampli op, pll, vco, multiplieur, ...) ainsi que les documentations techniques associées.

En outre, les élèves peuvent utiliser les appareils «classiques » d'un labo d'électronique (alimentations, multimètres, GBF, oscilloscopes, analyseurs de spectre, ...) ainsi que le logiciel de CAO «Cadence» qui leur permet de simuler leur montage.

\section{Un exemple de réalisation}

Les photos ci-dessous présentent une version aboutie de ce système.

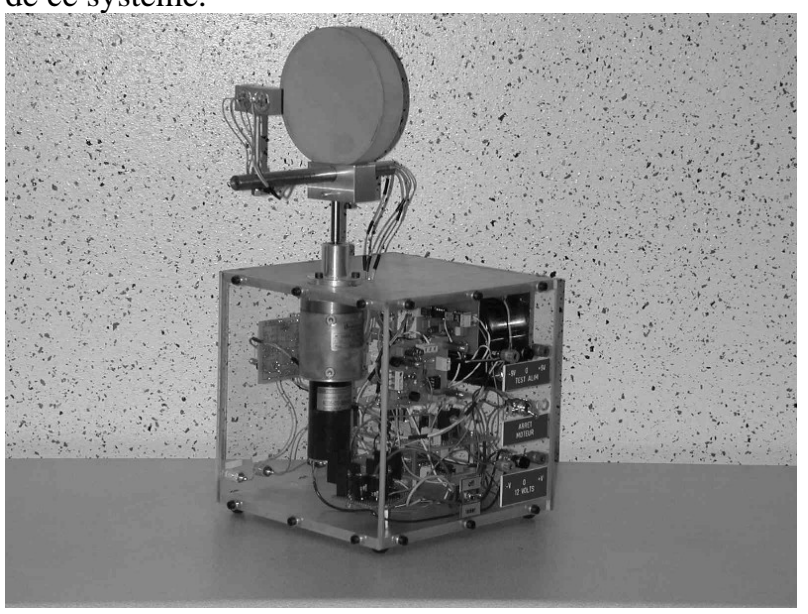

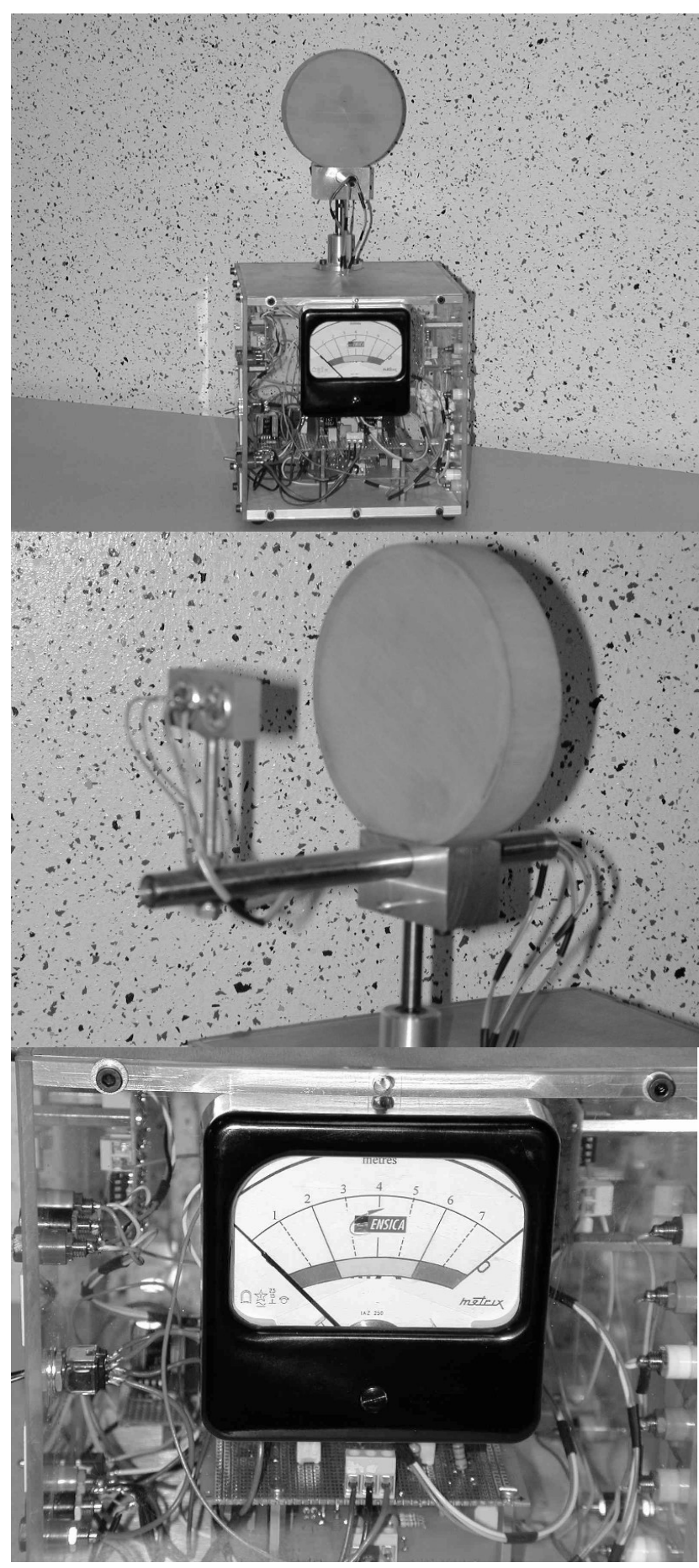

\section{Conclusion}

Ce projet permet de décloisonner les différentes disciplines formant l'enseignement EEA et de montrer, de façon ludique, comment fonctionne un système réel de RADAR de poursuite. Contrairement aux Travaux Pratiques classiques, ce projet laisse une part importante d'initiative aux étudiants et essaye d'être représentatif de la démarche que doit avoir un ingénieur. 